\title{
Transient peristaltic transport of grains in a liquid
}

\author{
Marco Marconati ${ }^{1} \star$, Sharvari Raut ${ }^{1}$, Farshad Charkhi ${ }^{1}$, Adam Burbidge $^{2}$, Jan Engmann ${ }^{2}$, and Marco Ramaioli ${ }^{1}$ \\ ${ }^{1}$ Department of Chemical and Process Engineering Faculty of Engineering and Physical Sciences, University of Surrey, Guildford \\ GU2 $7 X H$, UK \\ ${ }^{2}$ Nestlé Research Centre, Route du Jorat 57, 1000 Lausanne 26, Switzerland
}

\begin{abstract}
Pumping suspensions and pastes has always been a significant technological challenge in a number of industrial applications ranging from food processing to mining. Peristaltic pumps have become popular to pump and/or dose complex fluids, due to their robustness. During the transport of suspensions with peristaltic pumps, clogging issues may arise, particularly during transient operations. That is a matter of particular concern whenever the pumping device is used intermittently to generate flow only on demand. Further understanding of the transient dynamics of such systems and of the conditions that can lead to jamming would result in more robust peristaltic pump design. To achieve these goals, an experimental setup that simplifies the statorrotor assembly of a peristaltic hose pump was used. In this setup, a roller transfers momentum to a liquid suspension, upon application of a constant load. The evolution of the velocity of the roller was recorded for different concentrations of mono-dispersed spheres of different diameters. The flow is found not to be strongly dependent on the dispersed particle volume fraction, if the size of the suspended phase is comparable with the hose diameter. Conversely, the flow is strongly slowed down when their size is small and the particle concentration is increased. These findings could help improving the design of peristaltic pumps by a more appropriate sizing, given the diameter of the hose and that of the particles to be transported.
\end{abstract}

\section{Introduction}

Peristalsis is defined as the process of progressive contraction and relaxation of membrane that results in the forward net motion of a liquid contained in the membrane. Peristalsis is effective in transporting complex fluids and peristaltic transport is used by nature e.g. the human gastro intestinal tract and reproductive system. In peristaltic transport also solid particles can be moved by the progressive squeezing action at the walls. Physiological examples include transport of kidney stones through the ureter, embryo movement in the uterus, and swallowing of hard objects such as nuts, grains and pills. A number of engineering applications of peristaltic flow are commonly found in the medical field, wastewater treatment, food and pharmaceutical industries, cement, and mining. One basic design comprises a drive-coupled rotor with mounted shoes or rollers, which continually compress and occlude some portion of an elastic tube. This action moves the fluid through the hose providing a precise volume of fluid pumped. That makes these pumps extremely attractive for metering purposes, especially in applications where the highest levels of hygiene have to be ensured. With the right choice of elastomer, hose pumps can cope with corrosive and hazardous fluids without the need for using valuable materials and special alloys in their construction. The increased popularity of peristaltic pumps is also

\footnotetext{
^e-mail: m.marconati@surrey.ac.uk
}

due to other noticeable advantages in respect of classical positive displacement pumping systems: hose pumps are self-priming, reversible, and can cope with concentrated slurries (up to $70 \% \mathrm{w} / \mathrm{W}$ solids [1]). Moreover, peristaltic pumps are also suitable to transport shear sensitive fluids, due to the low shear applied on the fluid. Over the past years, a number of studies have considered numerical simulations of two-dimensional peristaltic flows both in finite and infinite domains solving the Navier-Stokes equation of motion in term of stream functions mainly retaining the approximations of low-Reynolds number and long wavelengths (lubrication theory). Characteristic phenomena of trapping and retrograde flow have been widely discussed [2] [8]. Additionally, several studies were conducted to understand the flow pattern in presence of dispersed solids [6][7][8][10]. The works of Fung [5], Hung [6], and Srivastava [7] constitute fundamental steps in the experimental investigation of peristaltic transport of solids. Numerical studies followed either using a one way coupling in which the fluid is treated as Eulerian and the particle motion as Lagrangian, or considering the full fluid-solid interaction between the suspended and the suspending phases [8] [11]. Despite the relevancy of those studies and the match with the corresponding experiments, there is still a significant lack of understanding the transient flow at startup. Furthermore, the common assumption of geometrical symmetry is questionable, as significant variations in the flow pattern arise when considering different ampli- 
tudes for the top and bottom walls [9] [2]. Depending on the number of rollers and the hose radius of curvature, an asymmetrical laminar wake, that generates an internal flow recirculation, can occur during the pumping cycle. In presence of suspended solids these recirculation could reduce the homogeneity of the product. Even worse, the presence of rigid objects and particles could clog the hose during start-up. The present study proposes a model, using a simplified hose pump design, to quantify the effect of both particle concentration and size on the measured angular velocity of a semisolid liquid.

\section{Materials and Methods}

The experimental setup consists of a circular bearing housing containing a thin plastic membrane that can freely deform under the action of a roller driven by a calibrated weight. The plastic membrane contains the liquid and the grains. The center of curvature of the bearing is coaxial with the shaft that supports the roller (Fig. 1). The supporting arm is balanced by a counterweight and the rotational inertia of the system was previously evaluated via free spinning of the arm. In order to investigate the most stringent conditions for peristaltic transport, a total occlusion configuration is used. The liquid volume used in the experiments was $6 \mathrm{~mL}$. The solid phase consisted of rigid mono-dispersed spherical inclusions, whose diameter spanned between $10 / 12$ and $5 / 12$ of the tube maximum cross sectional size. In the particular geometry considered by the study, the ratio of radius of the plastic tube to liquid length $(\alpha=\pi R / \lambda)$ is below 0.35 , while the maximum value of the Reynolds number, computed by $R e=$ $0.5 R c \alpha / v$ is lower than 1 . The density of the liquid phase is $1250 \pm 10 \mathrm{~kg} \mathrm{~m}^{-3}$, while the suspended phase density, measured with gas pycnomety, is $880 \pm 40 \mathrm{~kg} \mathrm{~m}^{-3}$. The liquid carrier consisted of $99.8 \%$ pure glycerol (SigmaAldrich, CAS Number 56-81-5) whose viscosity at $22^{\circ} \mathrm{C}$ was measured in compliance to ISO standard 3219 using a Paar Physica rotary rheometer (Model UDS 200, Paar Physica, Germany). Ambient temperature of $22 \pm 1^{\circ} \mathrm{C}$ was constant throughout the duration of the experimental campaign. The experiments were first performed without grains, using three different applied loads: $200 \mathrm{~g}, 270 \mathrm{~g}$ and $400 \mathrm{~g}$ (roughly correspondent to 2, 2.7 and $4 \mathrm{~N}$ ) to drive the roller. Then, solids were incorporated into the thick Newtonian liquid carrier at a volume fraction $\phi_{v}$ of either 0.06 or 0.12 . The experiments were performed with different sizes of beads, adjusting the number of beads to match the desired volume fraction. The experiment is triggered by the instantaneous release of a pin. The motion of the apparatus and the liquid is recorded with a fast camera at 150 frames per second. The roller is free to move and accelerate till it reaches a final angle of $165^{\circ}$ from the horizontal axis, where it is halted by a stopper. The suspension is instead free to exit from the tube. The movies recorded are used to extract the evolution of the position of the roller and the characteristic shape of the liquid through image analysis.

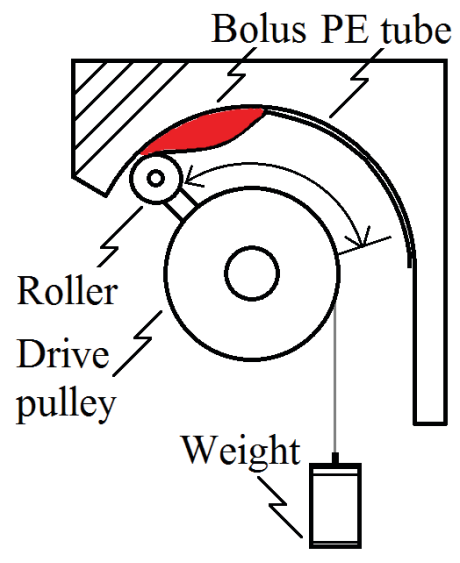

Figure 1. The experimental setup consist of a roller, driven by a set of calibrated masses, imposing a peristaltic deformation onto a thin plastic tube containing the liquid and the beads.

\subsection{Theory}

A simple mathematic model of the system, based on force balance was previously developed by Hayoun et al. The description of the drag force due to viscous dissipation was there derived using a Hagen-Poiseuille approach [12]. That solution well-matched the experimental results in the case of pure Newtonian liquids. Later, Mowlavi et al. extended the investigation to shear thinning liquids [13]. In their model, the drag force can be described by the mathematical expression presented in Eq. 1, in which the liquid bolus length and linear velocity are respectively $L$ and $\omega R_{A}, \mu_{0}$ is the dynamic viscosity of the liquid, and $\beta$ is a correction factor whose value is independent on liquid viscosity and driving force applied to the system and was experimentally determined by Mowlavi et al. [13].

$$
F_{d}=\beta 8 \mu_{0} L\left(\omega R_{A}\right)
$$

The above drag force was included in the differential equation of motion of the roller. The rotational parameters of the mechanical setup, such as the inertia of the rotating assembly and the friction of the bearing are characterized once for all with empty runs of the device. An additional correction is introduced to take into account the friction of the roller with the plastic membrane thereby the effective driving force momentum is consistently reduced by that fixed amount [13]. As a first approximation the dynamic effect due to the presence of suspended particles, of volume fraction $\phi$, was modeled considering a correction term to the viscosity of the liquid carrier $\left(\mu_{0}\right)$ obtained from the Krieger-Dougherty law for spherical particles, where the packing ratio $\phi_{m}$ was considered that of a simple facecentered cube arrangement of particles (Eq. 2).

$$
\mu_{\phi \neq 0}=\mu_{0}\left(1-\frac{\phi}{\phi_{m}}\right)^{-2.5 \phi_{m}}
$$

This approach, although simplistic in term of the actual particle-fluid and particle-particle interactions occurring for the geometries of field of motion considered, can 


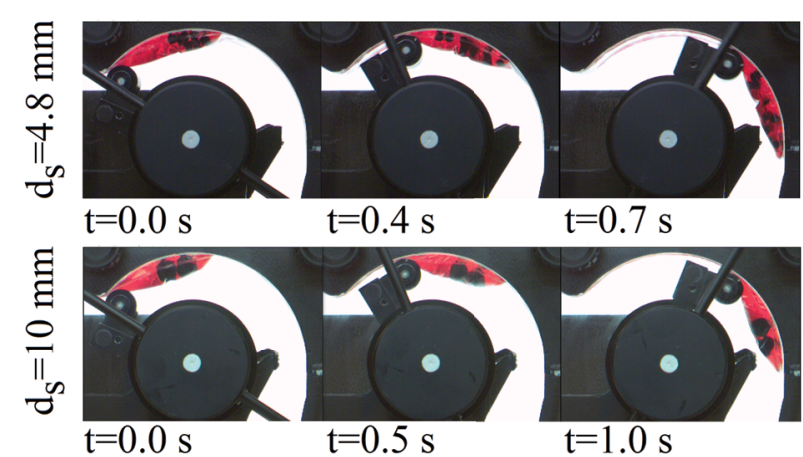

Figure 2. Screenshot taken at identical roller angular position in experiments at low applied load (200 g), with polyethylene beads of $d_{S}=4.8 \mathrm{~mm}($ at $\phi=0.06 \mathrm{v} / \mathrm{V})$, and $d_{S}=10 \mathrm{~mm}(\phi=0.12 \mathrm{v} / \mathrm{V})$.

still give useful insights to understand the experimental results.

\section{Results}

A visual comparison of the effect of inclusion size and volume fraction is reported in Fig. 2. Two extreme conditions of particle size and volume fraction are illustrated as an example, in experiments at low applied load (approximately $2 \mathrm{~N}$ ). The screenshots show the same roller angle positions, in order to appreciate the different dynamics from the time. The roller movement evolves in a timescale on the order of $1 \mathrm{~s}$ in the case of the biggest inclusion at the highest volume fraction of solids $\left(d_{S}=10\right.$ $\mathrm{mm}$ and $\left.\phi_{v}=0.12\right)$. The same force applied to a liquid with the lowest solid concentration $(0.06 \mathrm{v} / \mathrm{V})$ resulted in a $30 \%$ decrease in the transit time required for the solidliquid dispersion to reach the end of the plastic membrane in which it is initially contained. The information retrieved from a direct comparison of the screenshots of the videos is made more quantitative using image analysis tools. The roller position was therefore tracked so that the instantaneous velocity profiles can be plotted and more efficiently compared.

A set of experiments were conducted in absence of solid inclusions $(\phi=0)$ in order to validate the drag force model and obtain some reference lines for the subsequent trials with dispersed solids. Mean roller angular velocity profiles, together with the theoretical lines obtained with a viscous dissipation expressed in the form of Eq. 1, are illustrated in Fig. 3. The good agreement with the theoretical profiles is there evident comparing the small distance between the data points and this proved to be consistent with the results of Hayoun et al. [12]. Later, experiments were run in presence of suspended solid particles. In this case, velocity profiles highlighted an expected reduction in roller angular velocity when the volumetric fraction of particles was increased, consistently to the study by Lonzano [14], who considered a total occluded linear peristaltic pumping system for which $R e=50, \alpha=0.16$ and the ratio of solid inclusion diameter to nominal tube diameter was $1 / 3$. The decrease in angular velocity with

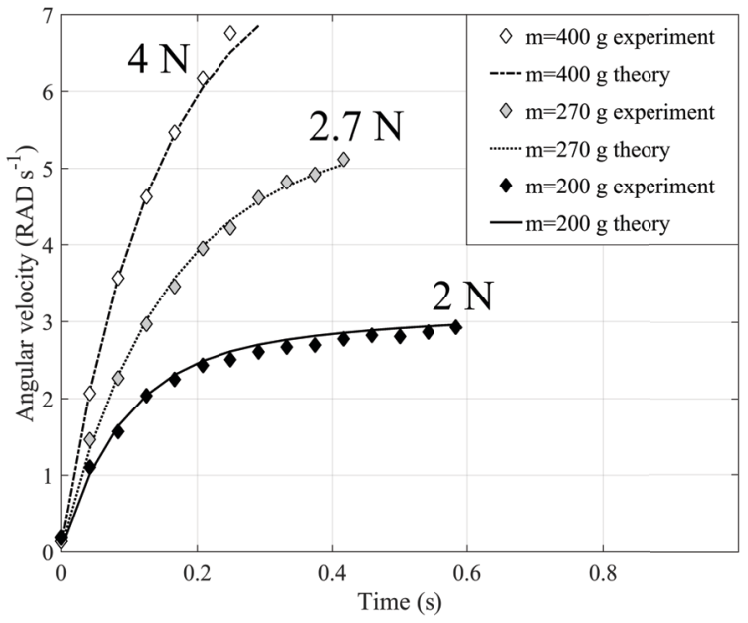

Figure 3. Experimental and theoretical roller angular velocity profiles for the three applied loads (200 g, $270 \mathrm{~g}$, and $400 \mathrm{~g}$ ) to the drive the roller, in absence of suspended solids $(\phi=0.00$ $\mathrm{v} / \mathrm{V})$.

increasing the volume fraction of suspended particles is however not linear. Our experimental results suggest that the effect depends both on the applied force to the system and on the size of the suspended particles. A comparison of the average velocity profiles obtained over two repetitions, for different concentrations, applied loads, and grain size, is reported in Fig. 4. Driving the system with a low applied force results in velocity profiles reaching quickly a steady state where the viscous dissipation equilibrates the imposed driving force. This kinematic condition is slightly affected by size of the suspended particles. A higher volume fraction accentuates the viscous dissipation and leads to a lower steady state velocity and a faster onset of the steady state.

Increasing the applied force results always in an increase of angular velocity, but the system takes longer to approach a steady state flow, as the steadily increasing velocity profiles obtained at the highest load configuration demonstrate. It can also be noted that the experimental profiles obtained at the highest load configuration are significantly closer to the theoretical lines drawn considering the increase in viscosity predicted with the KriegerDougherty law. Accordingly, the increase in viscosity, compared to that of the suspending carrier, is of the order of $15 \%$ in the case of the lowest concentration of suspended particles, and $35 \%$ for the highest volume fraction of grains. From Fig. 4 it is also clear that increasing the suspended particle volume fraction affects more the flow of smaller inclusions. Indeed, the velocity profiles for the $10 \mathrm{~mm}$ particles are not significantly different when the solid volume is doubled in the same suspending liquid carrier. Finally, the transport of biggest inclusion led generally to lower angular velocity. Although this effect is not strong in the range of inclusion size here considered, it appears consistently in the whole range of applied force considered. The simple model used still captures the ex- 


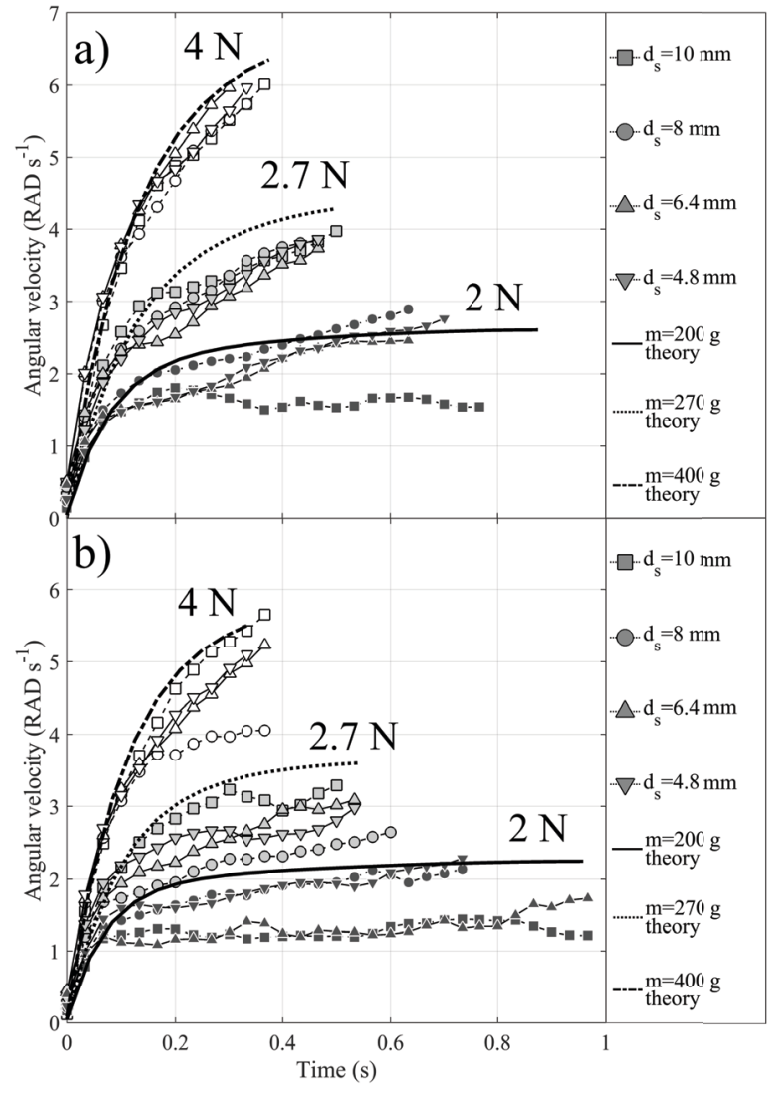

Figure 4. Experimental and theoretical roller angular velocity profiles at different applied loads to the drive pulley, in presence of suspended particles of diameters ranging from 4.8 to $10 \mathrm{~mm}$. The volume fraction of solids is a) $\phi=0.06$. and b) $\phi=0.12$.

perimental data points in the first phase of inertial regime, proving the correct choice of the rotational dynamics parameters chosen, and correctly individuates the transition to the viscous regime. The viscous dissipation force is however always under-predicted as the experimental angular velocity data point fall below the corresponding theoretical profiles, suggesting the relevant role of particlewall and particle-fluid interaction in this type of flow, even in the case of the smallest size of grains considered by the study.

\section{Conclusions}

This study aimed at investigating the transient start-up of a peristaltic pump transporting a thick Newtonian liquid with suspended hard grains. The experimental results showed that the roller velocity varies significantly as the concentration of suspended solids is increased, in particular in the case of smaller inclusions. Moreover, a significant reduction in pumping speed is always observed comparing the experimental results to the theoretical profiles plotted considering the apparent increase in viscosity predicted using a simple Krieger-Dougherty model. The effect of varying the inclusion size is stronger at lower applied loads, a condition in which the system moves quickly into a steady state flow. These results suggest the existence of a significant interaction of the suspended phase with the continuous liquid phase and highlight the need for developing a theory to describe the transient flow occurring during the start-up of a peristaltic pump in presence of suspended solids. Such theory would help designing robust pumps capable of performing precision metering of suspensions at different concentrations, regardless of the particle size being transported.

\section{References}

[1] B. Nesbitt, Handbook of Pumps and Pumping (Elsevier, Cambridge, UK, 2006) 38-39.

[2] M. Y. Jaffrin and A. H. Shapiro, Ann. Rev. Fluid Mech. 3, 13-36 (1971).

[3] J. C. Burns, T. Parkes, J. Fluid Mech. 29, 731-748 (1967).

[4] S. L. Weinberg, E. C. Eckstein, A. H. Shapiro, J. Fluid Mech. 49, 461-479 (1971).

[5] Y. C. Fung, C. S. Yih, J. Fluid Mech. 47, 93-112 (1971).

[6] T. K. Hung, T. D. Brown, J. Fluid Mech. 73, 77-96 (1976).

[7] L. M. Srivastava and V. P. Srivastava, J. Biomech. Eng. 111, 157-165 (1989).

[8] J. Jiménez-Lozano, M. Sen, P. F. Dunn, Phys. Rev. 79, (2009) 041901-9.

[9] L. Fauci, Comput. Fluids 21, 583-598 (1992).

[10] D. Takagi, N. J. Balmforth, J. Fluid Mech. 672, 219244 (2011).

[11] J. Jiménez-Lozano and M. Sen, Phys. Fluids 22, 043303-10 (2010).

[12] P. Hayoun, J. Engmann, S. Mowlavi, B. Le Reverend, A. Burbidge, M. Ramaioli, J Biomech. 48, 39223928 (2015).

[13] S. Mowlavi, J. Engmann, A. Burbidge, R. Lloyd, P. Hayoun, B. Le Reverend, M. Ramaioli, J Biomech. 49, 3788-3795 (2016).

[14] J. Jiménez-Lozano, Peristaltic flow with application to uretral biomechanics (University of Notre Dame, Notre Dame, IN, 2009) 158-164. 